\title{
The Interaction Space of a Multi-Device, Multi-User Music Experience
}

\author{
Henrik Sørensen \\ Aalborg University, \\ Department of Computer Science \\ Selma Lagerlöfs Vej 300, DK-9220 \\ Aalborg, Denmark \\ hesor@cs.aau.dk
}

\author{
Jesper Kjeldskov \\ Aalborg University, \\ Department of Computer Science \\ Selma Lagerlöfs Vej 300, DK-9220 \\ Aalborg, Denmark \\ jesper@cs.aau.dk
}

\begin{abstract}
The increasing interoperability between electronic devices in our everyday life offers great opportunities for ubiquitous computing in non-work settings. There are however aspects of the interaction space of interconnected devices, that we do not yet fully understand. This prohibits us from utilizing the full potential of the devices and the digital ecosystems emerging around them. We have explored the interaction space created around multi-device systems in a non-work setting, by developing a functional prototype of a multi-device music player and evaluating it in three different real-life contexts. The evaluations had a total running time of 12 hours and involved approximately 60 testpersons. Qualitative results collected throughout the evaluations provide insight into issues regarding interaction design of multi-device systems with multiple simultaneous users. Through a discussion of the results we point out areas of interest and design issues, revealed during the evaluations.
\end{abstract}

\section{Author Keywords}

Digital ecosystems; ubiquitous computing; distributed interfaces; music player; interaction design

\section{ACM Classification Keywords}

H.5.2 User Interfaces: Graphical user interfaces (GUI)

\section{General Terms \\ Design; Experimentation; Human Factors}

\section{INTRODUCTION}

An increasing number of digital devices are finding their way into our homes. Facilitated by standards such as UPnP [10] and initiatives like DLNA [5], they are gradually obtaining increased interoperability. These emerging networks of devices are no longer limited to desktop or laptop computers, but can additionally encompass devices like TVs, gaming consoles, smartphones, tablets, stereos etc. Such ubiquitous computing environments, also known

Permission to make digital or hard copies of all or part of this work for personal or classroom use is granted without fee provided that copies are not made or distributed for profit or commercial advantage and that copies bear this notice and the full citation on the first page. To copy otherwise, or republish, to post on servers or to redistribute to lists, requires prior specific permission and/or a fee.

NordiCHI '12, October 14-17, 2012 Copenhagen, Denmark

Copyright 2012 ACM 978-1-4503-1482-4/12/10... \$15.00” as digital ecosystems [15], opens up for new opportunities when it comes to media consumption. Broadband Internet connections and wireless network technologies provide the needed platform to support systems spanning multiple devices. Interoperability in current consumer products is however widely focused on data sharing and systems, distributed onto several devices, are often developed as adaptations of the same system and not as a complete system spanning across devices. One reason is, that most of these devices have evolved into network capable devices and are thus not inherently designed for multi-device interoperability[18]. Attempts of multi-device interfaces are often limited to a remote control metaphor for media players. Effort is therefore needed to uncover the full potential of the emerging digital ecosystems, from an HCI point of view.

The end goal is not to do uniform user interface design suitable for an array of devices. Such areas of study are primarily concerned with development efficiency. Instead we aim to inform interaction design which creates synergy by transcending boundaries and take device-specific strengths and weaknesses, as well as inter-device relations, into account. In order to do that we need to understand the challenges introduced when an interface is no longer isolated on a single device. Which attributes are specifically important for this class of systems? What implications does the distribution of the interface have for the users? What is the significance of the physical space? Multi-device systems furthermore offer the opportunity to allow several co-located users to interact simultaneously. This opens up for a potential to support social interaction through technology. It does however also add an extra layer of complexity that we need to take into account and understand, if we are to design usable systems. These dimensions of user interaction, social interaction and the physical space is what is encompassed by the term interaction space.

This paper uses the case of listening to music together, to explore the interaction space. First, some of the relevant work is presented, both regarding areas of multi-device interaction in general and media interaction. We then describe a prototype implementation of a multi-device music player, called MEET, that enables control from several users simultaneously. This is followed by a presentation of three field evaluations and the results gained. We then discuss the results, point out what future work they can encourage and lastly we conclude the study. 


\section{RELATED WORK}

This section places our work in relation to different topics in multi-device interaction and media interaction. It describes previous work in these areas and places this study in relation to it. The selected areas are relevant to this study in matters of scale, composition of devices, coupled display environments, domain and understanding digital ecosystems.

The focus of this paper is aimed towards exploring the interaction space of interfaces distributed onto multiple devices and obtaining an understanding of their role in digital ecosystems from an HCI point of view. One factor that greatly influences the interaction with multi-device systems is the scale of the interaction space. Studies like [9] operates in an urban environment, where the interface is distributed onto a number of personal mobile devices and public situated displays. The idea of having dedicated distributed user interface parts, for different devices, is the same as in this study. The scale however, creates another use situation and interaction environment where interaction can be more transient. Although the digital ecosystems are not limited to a specific confined space, the experiments in this study specifically explores the interaction space that is created around a multi-device system with co-located users.

Part of the previous work on distributed interfaces focuses on end-user composition of smart artifacts. Results from one study suggests that users see possibilities in either coupling smart artifacts present in their homes, or improving other household appliances by coupling them with input/output from other artifacts [3]. OSCAR is a technical approach to facilitate such composition of smart artifacts [16]. Even though our study similarly operates in non-work environments, the focus is different and aimed towards the actual interaction design of the systems that are either designed with a particular setup in mind, or the result of such end-user composition. It works on the assumption that a lot of the dynamics and rules of the interaction apply to multi-device systems in both cases.

Other studies specifically investigate the relationship between displays in coupled display environments [1][11]. This issue is very important to the system facilitating this study, where a shared situated display, a tablet and smartphones have to provide input and output simultaneously. Different studies particularly looks at either attention [2] or user performance [6] issues introduced when interfaces are distributed onto a combination of large public and small private displays. One common approach is to use the small touch enabled display for input only, like in [14], facilitating a direct replacement for mouse/keyboard input. This approach is very generic and applicable for a variety of applications. In this study however, we investigate an approach where both input and output is distributed.

Various studies have been aimed towards specific trends in interaction design for multi-device environments. One study presents a conceptual framework for design of collaborative multi-device systems [4]. They focus on multi-device web-browsing and evaluates a prototype of a developed architecture. Another study focuses on different modalities [20]. Through the development and evaluation of a multimodal media center interface, they explore the different input modalities, speech, physical touch and gestures. The work of Greenberg et al. [7] uses proxemics [8] to create multi-device systems, which are aware of spatial relations between users and devices. Despite the wide array of directions, they all share the common goal of contributing to a better understanding of the ubiquitous digital ecosystems that emerge through the interoperability between devices.

The specific domain, used as a case for this study, is listening to music together. The concept for the distributed music control is influenced by the work of O'Hara et al. on the music system Jukola [17]. Jukola distributes control onto separate devices using nominations and votes to create a democratic music player in public space, which was tested in a bar environment. A situated touch screen is used to perform nominations and PDAs are used to vote for nominated songs. PartyVote [19] is another system that similarly provides a democratic music jukebox, but focuses on private social gatherings and minimal interaction. The interface is centralized and divided into a selection window and a visualization window. UbiRockMachine is a music player that offers the same kind of multi-device interaction, but with the purpose of distributing music from un-signed music producers to people in shared urban spaces [13]. An important aspect of MEET, used in this study, is that the interface is distributed to the users own devices, enabling them to use it as an access token to share music, as well as providing a familiar interaction device. We used the music system as means of further studying the interaction spaces in digital ecosystems and not particularly the music domain.

\section{CONCEPT AND ARCHITECTURE}

MEET is a music player aimed at enhancing the experience of listening to music together in a social context. The idea of MEET is to enable several users to influence the music on two levels: First of all, to allow users to share their personal digital music collection. Secondly, to allow the group of users to control playback through a distributed interface. For the playback control, a system is implemented where the underlying idea is conceptually similar to that of, e.g., Jukola [17]. Interaction is thus spread across several devices each playing their distinct role. The concept development is the result of a technology analysis, where unexplored concepts in current systems were identified, followed by a design workshop. One result of the workshop is a set of overall guidelines for the system interaction:

- The music never stops: Songs are never interrupted and there is always a song ready to be played next.

- The system is secondary: MEET is not designed to be the center of attention. It is a music system playing in the background, rather than a multi-user entertainment system.

- Different levels of participation: It should be possible to influence the music on different levels. A high level of participation should be rewarded but, in order to keep the system secondary, it should also be possible to use the system with less effort. 


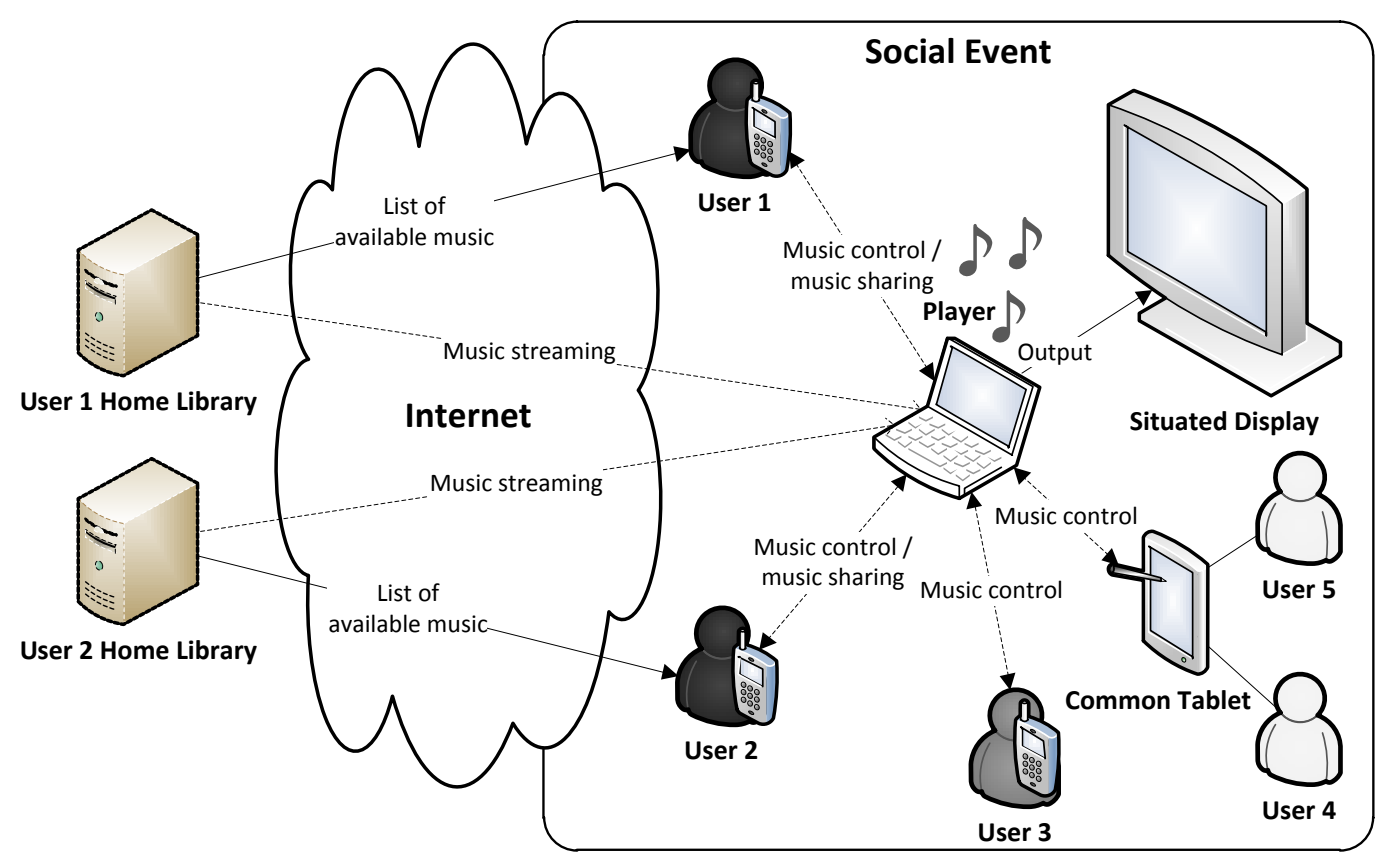

Figure 1: The architecture of the system depicting the relations between the different system entities.

An important aspect is to avoid the need to keep portable devices synchronized with the music collection at home, in order to access this music elsewhere. Instead MEET relies on streaming and an access-granting token in the form of the users personal smartphone. The access granting-token, being the most novel feature, lets the users connect to a player and share a selection of their music at home. Using personal smartphones create a feeling of bringing your music collection along, even though in reality it is only meta information about the music that needs to be transferred. It furthermore provides a very open approach to a multi-device system, where any user can walk up, connect and use his own smartphone as an interaction device. At the player a set of music is constituted where songs are streamed directly from each user's home libraries per demand.

The entire architecture can be seen in Figure 1, showing an example setup of users and devices. The system architecture comprises five different system entities:

1. A player, which is basically any computer running the player part of the software. This is the part responsible for maintaining the shared set of music and handle music playback. The player also handles connectivity to both handheld devices and home libraries. Besides the user interface shown on the situated display, the player also has a user interface primarily used to connect mobile devices.

2. A situated display which shows the primary user interface of the player and is shown on a large flatscreen TV or a projector. The situated display is the common output device for all users and provides information about nominations as well as the current song playing.
3. A home library for each user who wants to share music. The home library part of the software handles information about the chosen music from a user's personal digital music collection and makes it available for direct streaming to a player.

4. A smartphone application which both acts as an access-granting token and an interaction device for the distributed player control. The idea is that users who would want to share music, and/or participate in the the music control, connects their own smartphone to a player and uses it for this purpose.

5. A common tablet hosting a modified version of the smartphone application that is exclusively for nominating and voting. Its primary function is to allow users without a compatible smartphone to influence the music, but it also creates a physical interaction space around the system not constituted by the distributed nature of the interaction.

The home library is the most problematic part of the architecture and introduces a range of issues regarding availability. Even if people have a digital music collection, it is not likely that they have a computer running all day long. For the experiment in this study, the issue could be handled by setting up an accessible library, before the evaluation, for each user contributing music. In order to be useful in a broader sense, cloud storage services or commercial music sevices could however be considered as a replacement for the home library. Where the music is streamed from is more of a technical and legal issue than an HCI challenge. 


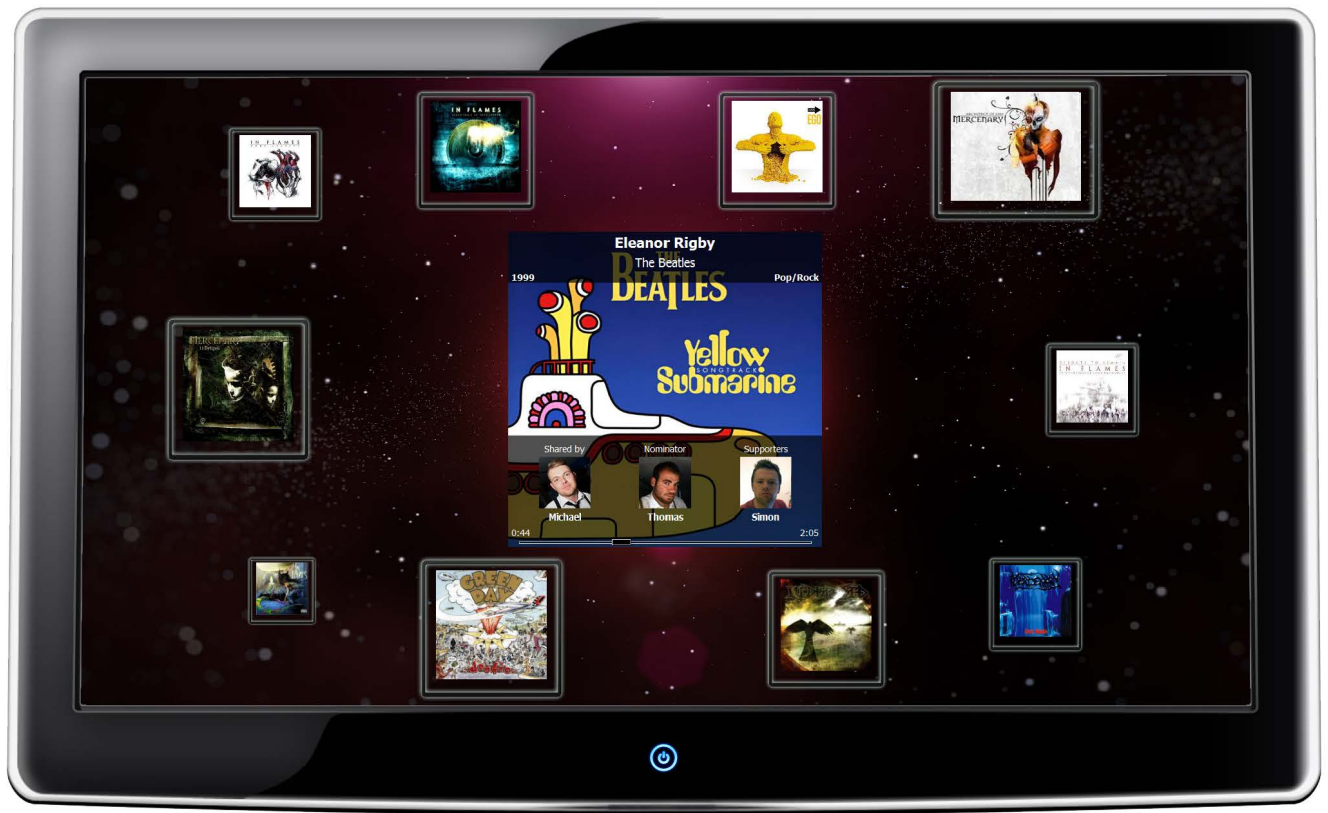

Figure 2: The situated display contains information about the current song playing in the center, surrounded by representations of nominated songs. The size of the nominations indicates current ranking, meaning that the largest nomination will be played next.

The design guideline of enabling different levels of participation and the nature of the setup, creates the notion of three types of active users from a device oriented point of view:

1. Users who have a running home library and a compatible smartphone, enabling them to both share music to the player and take part in the music control (Users 1 and 2 in Figure 1).

2. Users who do not have a running home library but have a compatible smartphone, enabling them to participate in music control (User 3 in Figure 1).

3. Users who do not have a compatible smartphone, who can use the common tablet as their control device (Users 4 and 5 in Figure 1).

It is important to note that there is no inherent conceptual limit to the number of users of each type. Although the system is designed with the first type of user in mind, the other two types of users create different interaction scenarios, which help uncover several other aspects of the interaction space.

\section{IMPLEMENTATION}

This section describes specifics about the implementation of the system used in the evaluations. This specifically covers a detailed description of the two major functionalities, nominating songs and voting, which include screenshots of the actual user interface. A description of the differences in the tablet interface is also provided. The implementation of MEET furthermore includes an identity and connection aspect which will not be covered by this paper.

\section{Overview}

The user interface of MEET is distributed across various devices which in turn is also distributed to several users. The situated display serves as the only common output device for the player. Although it does have an additional part of the user interface on a small screen, this is primarily used for connecting users to the system. All user input happens through the handheld devices. Output concerning individual actions, such as keeping track of nominations and votes, is provided directly on the handheld device and the current state of the system is summed up on the situated display (see Figure 2).

\section{Nominating Songs}

One of the primary functionalities of MEET is to enable users to browse the composite set of music and nominate songs. Nominating a song means that it will become a candidate for the next song to be played. It also means that it will be represented in one of the nomination slots of the situated display and be made available for voting. Nominations are placed randomly in one of the available slots and stays in the same position until it is either played or eliminated, to avoid confusing the users. A set of rules has been implemented to make the system comply with the concept guidelines. The system will always make sure that there are at least three songs nominated, by nominating random songs from the music set if the number drops below. This will ensure that there is always a song ready to be played and furthermore encourage interaction by always having songs to vote for.

Users nominate songs using their handheld devices. The interface for browsing the music set on the player is straight forward. Filtering is done through a four-level hierarchy where users can go through genres, artists or albums, tapping 


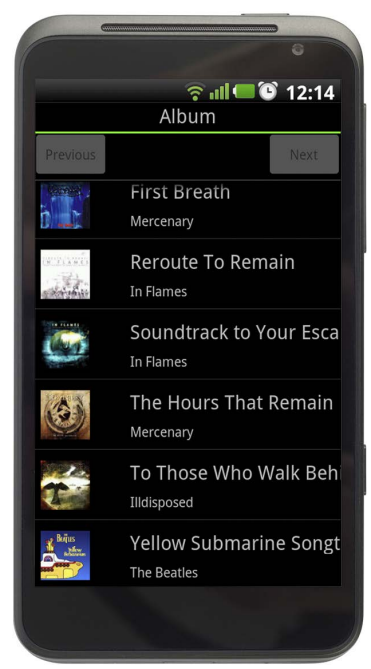

(a)

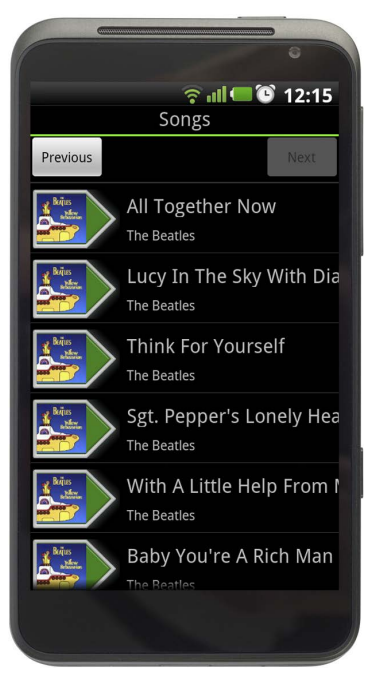

(b)
Figure 3: Nominating songs is done by browsing through the music set on the player using the handheld devices. The album view is shown in (a) as an example of the browsing levels. In (b) the song view is shown where an arrow is added to each element, indicating that it can be swiped to nominate.

to reach lower levels until they can choose from a list of songs corresponding to their choices. These songs can be nominated by swiping to the right (see Figure 3b). An additional feature instantly shows 15 random songs to pick from and can, for example, be used if a user is not looking for something in particular and wants to be inspired.

\section{Voting}

An important concept characteristic is that no person can single handedly decide what songs to hear, hence another primary functionality is to vote for nominated songs. Like the nominating functionality, voting is done using the handheld devices. The interface presents users to a list of the current nominations where they can choose to give a plus or minus vote for each nomination (see Figure 4). A plus or minus vote will simply add or subtract one point from a total score respectively and the song with the highest score will be played next. Additional elimination rules are implemented and are enforced at the end of each song, to make the system more dynamic. The first rule removes nominations with a negative total score. The second rule removes nominations that have not received votes after three songs.

Representations on the situated display change continuously according to votes, where size is used to visualize the current ranking. In order to maintain a consistent and structured layout, each nomination has a maximum and minimum size and individual sizes are computed relatively. A plus vote on a nomination is therefore not necessarily causing this particular representation to grow in size, but could instead make other nominations shrink. The sizes are however calculated on a pixel level meaning that no larger discrete intervals are defined. Feedback is provided on the smartphone application which will show a short notification confirming user input.

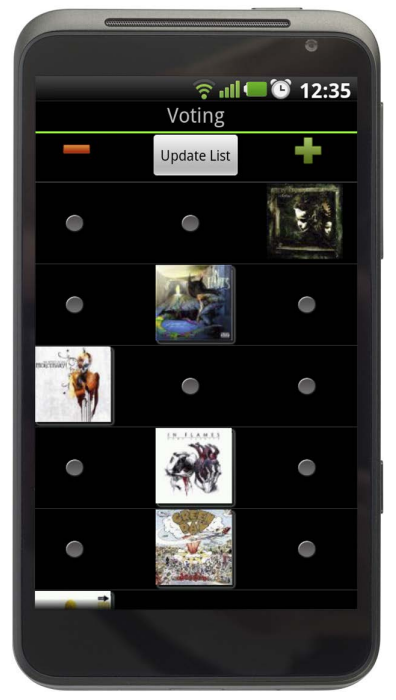

Figure 4: Voting is done from a list of the current nominations where each nomination can be given a plus or minus vote. The nominations are visually identical to the representations on the situated display.

\section{Tablet}

The tablet application is basically a limited version of the mobile application. It is a device with the primary purpose of letting users, without a compatible smartphone, nominate and vote for songs. An important difference between the mobile application and the tablet is the voting system. Because the mobile application only allows one vote per song it is not suitable for a common device. If the user had an account in the tablet application this could be possible but instead another voting system is implemented. Multiple votes for each song can be given, but there is a timer countdown after each vote, disabling voting for a short period of time. The goal was both to set a limit on total votes and furthermore to avoid a use, where one person would keep voting for a single song, giving an unfair advantage. The overall visual expression from the mobile application is kept intact besides some resizing.

\section{USER STUDY}

The ultimate purpose of MEET is to facilitate an exploratory study of the interaction space around interfaces distributed across several devices and where multiple users interact simultaneously. The user study is aimed at exploring the interaction space, in order to obtain a better understanding of the conditions and challenges, both regarding $\mathrm{HCI}$ and interpersonal relations, established by injecting such a system. This section describes the design of the study, including descriptions of the evaluation setups.

\section{Study Design}

For each evaluation, the prototype was set up on available hardware, as there are no mentionable hardware requirements and basically any desktop or laptop PC/Mac, with a stable broadband Internet connection can be used. The home library and player software is developed in Java to support various operating systems as well. The situated display for 
the standard prototype setup is a 42 " full HD flatscreen, but resolution can be configured to match specific output devices. The smartphone and tablet application is developed for Android and supports a wide array of various handsets and Android versions. The tablet used is a Creative Ziio 10, featuring a 10" resistive touchscreen and Android 2.2.

The study consists of three in-situ field evaluations which made it possible to explore the use of MEET in realistic settings. This was carried out by setting the system up at social events and letting people use the system as a direct replacement for what would otherwise be used to play music. Some participants had been contacted in advance in order for them to set up a home library that would form the basis for the available music. As a backup we provided a set of additional songs. Other participants were not introduced to the system in advance and would volunteer for participation at the event. As the study is exploratory in nature, the intention of having three evaluations is not to replicate the experiment, but rather to be able to try out the system in variations of the physical and contextual settings. The duration of the evaluations was dependent on the specific event.

Various data collection methods were used in the study. Observations was made during the evaluations where we would take note of any events regarding user behavior or direct comments about the system and system use from participants. After the system had been in use for a period of time we would furthermore start conducting semi-structured interviews. Only participants who was in some way interacting with the system was interviewed, but was otherwise randomly chosen. In addition to the two primary methods, video recordings and embedded data logging were used to support findings and as a review tool in the subsequent data analysis.

\section{Evaluation 1.}

The evaluation was conducted at a dormitory Friday bar ${ }^{1}$ where a stationary computer connected to a stereo is usually used as the music player. Previously a music library on the computer provided all of the music, but lately they have almost exclusively switched to online streaming services. The computer and stereo is placed behind the bar and the bartenders therefore normally act as DJs. The bar is however run by volunteers living at the dormitory and the music control is therefore very casual, where everyone can request songs and act as a DJ.

As seen in Figure 5, the Friday bar was equipped with a projector and a projection screen which was already placed strategically to make it visible from the entire room and was therefore suitable to act as the situated display. The tablet was placed in a corner near the situated display and the laptop running the actual player was placed behind the bar for connectivity reasons.

\footnotetext{
${ }^{1}$ The term Friday bar refers to a concept in Denmark, which is a non-commercial bar in relation to e.g. a workplace, educational institution, dormitory, apartment building etc. where people can socialize at the end of the week.
}

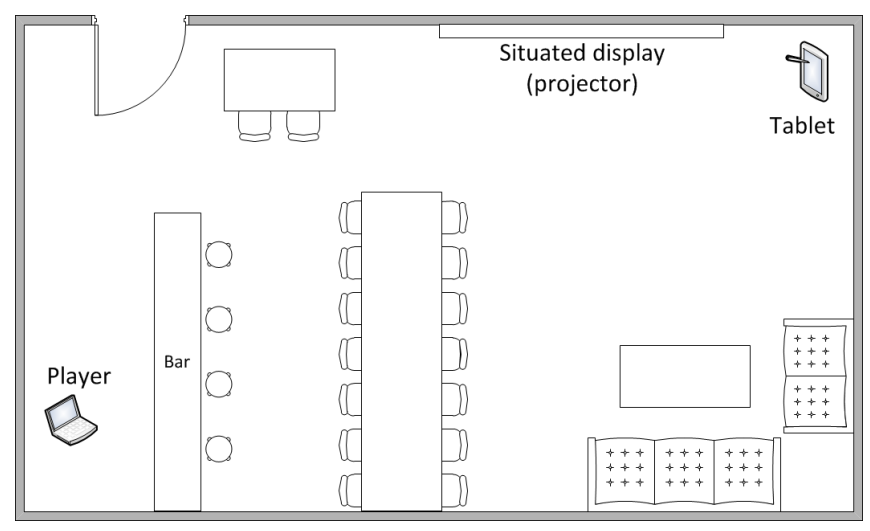

Figure 5: Floor plan of the location for evaluation 1.

During the evaluation a birthday party was held. Because the bar was still open as usual, the participants were a mix of people invited to the party and the bar's normal guests. Throughout the evaluation, the number of visitors in the bar varied, but as soon as the birthday party guests had arrived, it was constantly around 25. Participants were primarily students attending different educational institutions and the age range was 20-27.

\section{Evaluation 2.}

The location of the second evaluation was the Friday bar at the Department of Computer Science of Aalborg University. A laptop connected to a stereo is usually the music setup and free online services are frequently used to find songs not present in the music library of the laptop. Everyone can access the music player and no one is directly responsible for what is played.

The situated display was placed in a corner with visibility in mind. The tablet was placed on the table, in front of the TV, in a stand normally used for documents to make the tablet more visible and to create an explicit interaction space in front of the situated display. The player was placed on an adjacent table, which made the connectivity to the system very accessible to the users. The entire layout can be seen in Figure 6.

The bar is normally open to everyone, but the majority of guests is usually students and employees at the department. At the evaluation additional guests were invited with the purpose of evaluating the system. The number of participants was around 25 persons with an age distribution of 20-40. The system was tested predominantly by users with high IT competences.

\section{Evaluation 3.}

The social event for the third evaluation was arranged specifically to test the system. In addition we used the opportunity to perform participatory observations, in order to gain further insight into findings made at previous evaluations. The evaluation setup was a smaller party in a private apartment. The host usually uses a computer with iTunes as the music player, but sometimes online streaming services are used as well. 


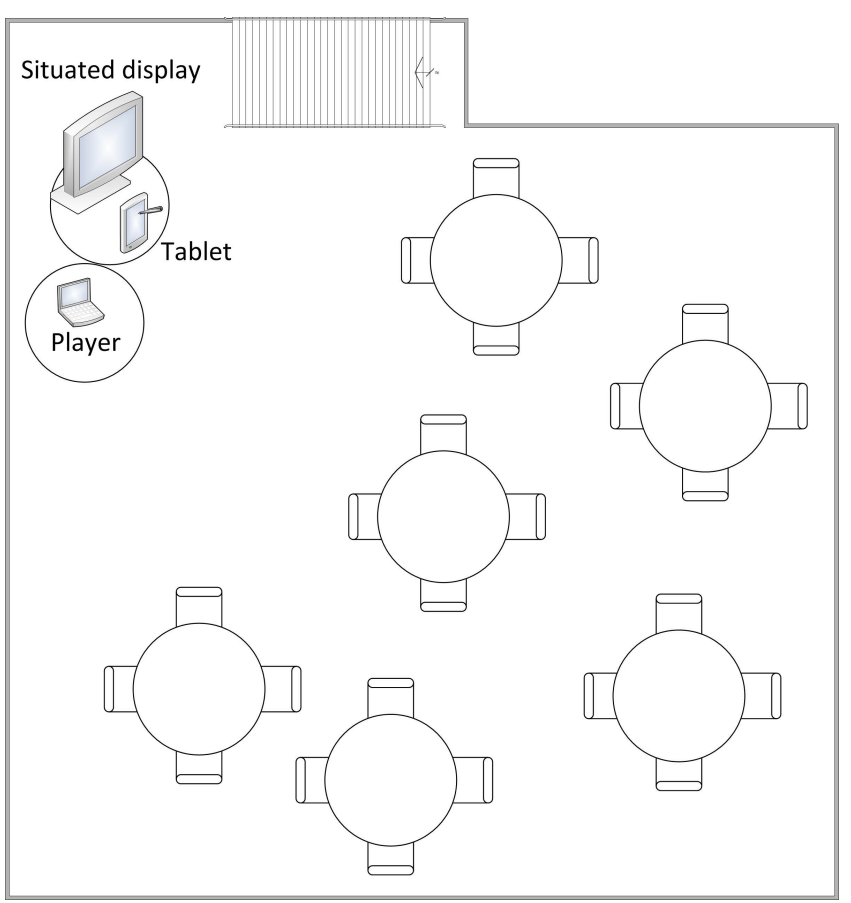

Figure 6: Floor plan of the location for evaluation 2.

For the situated display the existing living room TV was used and the laptop facilitating the player was placed next to it. The tablet was initially placed right next to the player but as it was a small private party and the atmosphere was less formal than at the two larger social events, the tablet was quickly removed from its initial placement and passed around. Most of the evening, people without an Android phone took turn keeping the tablet at their disposal. Figure 7 shows the layout of the room.

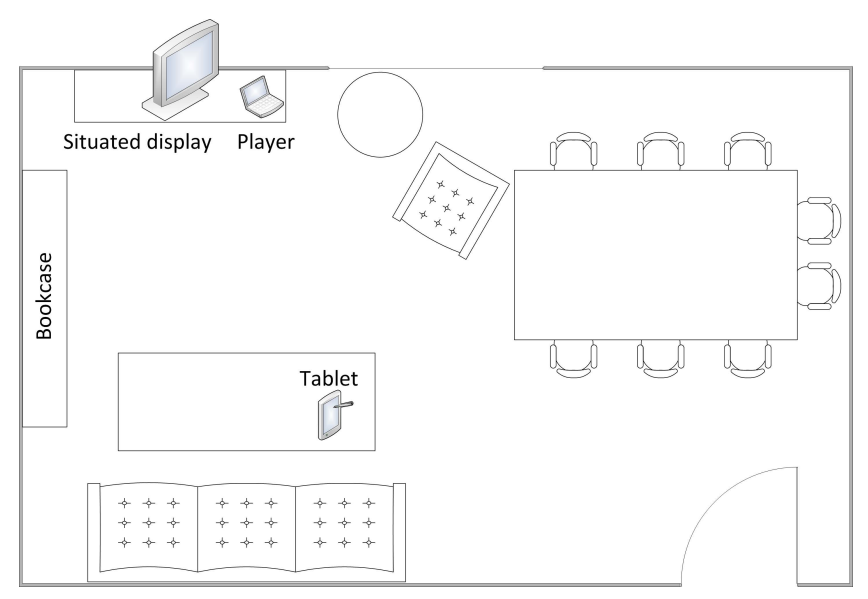

Figure 7: Floor plan of the location for evaluation 3.

The participants at this evaluation were students and former students at the Department of Computer Science, a lawyer, a pedagogue, a psychologist and two persons studying at the University College in Aalborg. The total number of users were 13 with an age range of 19-29.

\section{RESULTS}

In this section we initially present general results of the overall activity level during the three evaluations. Afterwards we go into detail on the qualitative results of the study. These have been categorized according to areas of interest identified during the evaluations and subsequent analysis. In some cases results are presented using quotes representative for user perceptions ${ }^{2}$.

\section{Overall Activity}

Table 1 shows data about interaction in each evaluation gathered from the embedded log system in MEET. We found that there was a general desire to take part in the negotiation about what music to listen to. Because the implementation of the access-token and control device was limited to Android devices, everyone was not able to participate directly though. Instead smaller groups would form around the tablet or participants with a compatible smartphone. Smartphones would also occasionally be passed around between participants. The log- data from system use therefore only encompass the direct interaction with the system, which still indicates a high level of activity.

\begin{tabular}{ccccc}
\hline Eval. ID & Duration & Connections & Nominations & Votes \\
\hline 1 & 4 hours & 10 & 81 & 258 \\
2 & 3 hours & 15 & 97 & 522 \\
3 & 5 hours & 8 & 164 & 915 \\
\hline
\end{tabular}

Table 1: Interaction with the system during the three evaluations. Connections are unique connected devices including the tablet.

A consistent observation was that the use of the system would not be evenly distributed over the course of any of the evaluations, due to several reasons. As expected, the novelty factor of the system created an increased activity every time new participants connected to the player, where they would spent time getting to know the concept and experiment with the different features. There would furthermore be periods with general highs and lows, typically caused by activities at the social event. The music currently playing would itself influence the activity level and participants would often be provoked to nominate or vote, if music they did not like kept "winning".

\section{Distribution of Control.}

The distributed control is one of the major features that separates MEET from conventional music players. Similar to experiences made in the study by O'Hara et al [17], we found that an important aspect to a lot of people is to feel involved in the process of choosing music. Users expressed satisfaction with the fact that they were able to obtain influence, even though they were given an indirect form of control compared to what they were used to. The fact that there was no direct way of changing songs was actually welcomed by the majority, as it would avoid some drawbacks of conventional music players in similar situations:

\footnotetext{
${ }^{2}$ Since evaluations were conducted in Denmark, all participants, with a few exceptions, were Danes. Quotes have therefore been translated into English where necessary.
} 


\section{"Nobody can suddenly come up and click and then the whole playlist is gone."}

Some participants specifically liked that the system created a situation, where no single person would be liable for selecting a song. People would not be concerned about nominating songs, as they would trust the system to ensure that songs were only played, if other people would want it to as well. They could just choose what they wanted and even select a number of songs they would like at the same time, without continuously getting accused of changing the music:

"Well, as long as it's democratic you can just choose what you feel like listening to and then the others can vote it up or down."

At the same time the participants would also generally accept that others might not have the same taste in music. Participants would often express satisfaction if one of their nominations "won", but we did not get a lot of negative comments about people not getting nominations played. In fact it seemed like people would get some instant satisfaction when nominating a song and then think less of whether or not it was played.

Having the nomination and voting distributed onto the handheld devices, makes it possible to influence the music without physically breaking away from the social context. This furthermore supported those who did not want to put themselves "out there" when they wanted to choose songs:

"Now, we don't know these people very well so... So we can just sit and vote at the party. Then we don't have to go up and discuss the music."

This might not be a desired effect, but it shows that there are certain social aspects which influence the use of the system and that the design of the system can have a strong influence on the social interaction.

\section{Multi-device Interaction.}

The interface is made up of both common and individual elements that allow for simultaneous interaction by multiple users. This gives rise to a lot of challenges that are both caused by the distribution of control, as well as the social aspects of the system use.

One interesting finding in particular was however caused by the fact, that we had not put much effort into streamlining the interaction on the hand-held devices. The most repeated comment from participants was that the process of nominating songs was too cumbersome:

\section{"I don't know why you don't just have it all under one [page] with a scroll bar at the right side. Then you don't have to go back all the time, you can just scroll up or down."}

As a direct effect, we observed that the amount of nominations throughout evaluations would gradually decline and the number of votes would incline. It is much faster to vote for a song than browse through the music set, to nominate a song, and participants would eventually prioritize socializing over system interaction, thereby giving up influence.

A result directly related to multi-device GUI design was found due to the underlying implementation of visual feedback on the situated display and the hand-held devices relatively. Whenever a user performs a vote or a nomination, the system will not update the user interface on the hand-held device before the player has registered the query, updated the situated display and sent a response accordingly. Users would wait for the mobile interface to update before turning their attention towards the situated display, thereby missing the results of their actions. This was consistent behavior through all three evaluations and the problem was more severe in evaluation 1 , where the tablet was not placed directly in front of the situated display.

Additional challenges are introduced when providing feedback to multiple users on a single situated display. In MEET there is no explicit feedback to specific users, besides what is shown on the mobile interface. Users would however often want to confirm that their actions were successful by looking at the situated display and this sometimes caused confusion, when several users were interacting from each their hand-held device simultaneously. Effects were further enhanced by the before-mentioned issues regarding coordination of feedback. A specific problem is that users misinterpret other users' actions as the system either not reacting to their own actions or believe that an error occurred.

The physical settings of each evaluation turned out to influence the system more than expected and some comments and observations were directly related to the physical environment. In some cases elements on the situated display was not fully visible to all participants, either due to objects blocking the line of sight or because of distance. Some participants expressed that it could be hard to tell if their actions were successful, but that they had to trust what they could see on their hand-held devices. This was primarily due to the strong dependency of the situated display caused by minimal feedback on the mobile device.The juxtaposition of the tablet and situated display was also crucial for the use of the tablet. When it was placed directly in front of the situated display, it created a interaction space not present in the other evaluations. The role of the tablet would also be very different, depending on whether it was positioned in a corner, in a place people would regularly walk past or occasionally be passed around .

\section{DISCUSSION AND FUTURE WORK}

In this section we discuss the findings made and identifies issues that would be interesting to pursue through future work.

Implications for social interaction. Comments from participants revealed that the nomination and voting system did not actually introduce a new dimension to the activity of listening to music in a social context. It rather provided a technical solution to some of the face-to-face negotiations that would otherwise occur about the music control. At the dormitory Friday bar, the bartenders would, e.g., normally 
be the ones responsible for handling song requests from guests and then decide when or if to accommodate their request. Discussions about what to hear would still arise during these evaluations, but would differ, as there is no singular point of control. People would instead gather around their smartphones, or the tablet, in smaller groups to discuss what to nominate and what to vote for. For some it would provide means of avoiding social interaction normally experienced in similar situations, as there is not necessarily any explicit signs telling that a person is interacting with the system. In the scenario of the evaluations the use of people's personal devices for a common purpose have shown to change parts of the social interaction. Not only does it relocate some responsibility from social interaction onto a technical solution, it also provides means to either anonymize or accentuate users in social contexts.

Distribution of functionality. The issues regarding the cumbersome browsing on the hand-held devices suggest that it plays an important role for the user, that the time needed to perform tasks is not significantly longer than they are used to in other systems. Time is especially important, as effort used on finding a song does not ensure that the song gets played. As it may seem like a trivial finding, the reasoning and relevance to multi-device interfaces is however more complex. In the case of multi-device systems, it is not only a question of designing the interfaces for the involved devices individually, but just as important to take the design of the system as a whole into account. As design improvements and added functionality on the mobile interface might reduce the issue in this situation, the question might be if the functionality should be on the hand-held devices in the first place? Browsing for songs is not implemented on mobile devices, because they are more suitable for the task, but because it provides mobility. The task is furthermore typically done on mobile devices in contexts different than what MEET is designed for. Work has been done addressing the issue, like in [12] where a gestural audio-tactile interface is used as an approach to handle music exploration in a mobile context. It is however important to consider which device is the most appropriate in most cases.

Coordination of interaction. The evaluations showed that when a user interface is distributed across several devices, it is crucial to be aware of the way feedback is applied across different devices, to give users a natural understanding of their actions. Fast response times are not necessarily a quality, in these systems, if feedback is not coordinated. This became apparent, during the study, due to issues regarding visual feedback and the difficulty of simultaneous visual attention. The difficulties of presenting feedback to multiple users on a single display is an additional challenge. However, the solutions might not be restricted to the coordination of feedback, but may also involve the type of feedback and interaction form. Different forms of feedback (tactile, auditory etc.) as well as different forms of interaction modalities could be considered for specific purposes, to accommodate some of the issues raised by the context and the environment.
Physical environment. Findings specifically provoked by differences in evaluation setups, indicate the significance of the physical environment to the interaction space of multi-device systems. These are primarily focused on visibility and availability of interaction devices. Part of the issues with MEET, is due to the dependency on visibility of the situated display. The collaboration between different devices introduces a lot of variables and one aspect that needs to be considered is dependencies the interface has to the physical environment it is placed in. As tailoring the design, of each multi-device system, to the physical surroundings is not a sustainable solution, other design solutions should be considered. One possibility is to use proxemic interaction [7], enabling multi-device systems to obtain context awareness and thereby be able to adapt to changes in, e.g., location or orientation of devices and users. Another aspect to consider is how less dependencies between visual elements on different devices can make the physical environment less significant.

\section{CONCLUSION}

This paper presented a field study exploring the interaction space created around a multi-device, multi-user system in non-work settings. By implementing a working prototype of a music player and conducting evaluations in different real-life environments, we have obtained greater insight into areas of concern, in relation to design of user interfaces distributed onto multiple devices with multiple simultaneous users. The field study was conducted through three in-situ evaluations with a total running time of 12 hours and approximately 60 test persons. Data have been collected primarily from a combination of observations and semi-structured interviews. Video recordings and embedded logging have been used to support findings in the data analysis.

What the study has illustrated, is especially the complexity introduced, when a user interface is distributed to multiple co-located devices and users. This both concerns the actual interaction design of the distributed interface and the role of such technology in a non-work environment. The identified areas of interest concern implications for social interaction, distribution of functionality, coordination of interaction and the significance of the physical environment.

Our key contribution is uncovering these specific areas of interest and the results provide insight into how they each influence the interaction space of multi-device, multi-user systems. Although the results are not exhaustive, they provide a starting point from where further research can be done. The issues found do not have trivial solutions, but directions from previous work might be applicable to lead us towards an understanding of how to design distributed interfaces for digital ecosystems.

\section{ACKNOWLEDGMENTS}

A special thanks goes to Liv Stahl Madsen and Simon Lind Damgaard for their work on the prototype development and initial study, as well as to Jeni Paay and Mikael B. Skov for their contribution on the design workshop. We furthermore thank all hosts and participants of our evaluations. 


\section{REFERENCES}

1. Cauchard, J. R., Löchtefeld, M., Irani, P., Schoening, J., Krüger, A., Fraser, M., and Subramanian, S. Visual separation in mobile multi-display environments. In Proceedings of the 24th annual ACM symposium on User interface software and technology, UIST '11, ACM (New York, NY, USA, 2011), 451-460.

2. Chorianopoulos, K., Fernández, F. J. B., Salcines, E. G., and de Castro Lozano, C. Delegating the visual interface between a tablet and a tv. In Proceedings of the International Conference on Advanced Visual Interfaces, AVI '10, ACM (New York, NY, USA, 2010), 418-418.

3. Coutaz, J., Fontaine, E., Mandran, N., and Demeure, A. About composing our own smart home. In Proceedings of the International Conference on Advanced Visual Interfaces, AVI '10, ACM (New York, NY, USA, 2010), 405-406.

4. Ding, Y., and Huber, J. Designing multi-user multi-device systems: an architecture for multi-browsing applications. In Proceedings of the 7th International Conference on Mobile and Ubiquitous Multimedia, MUM '08, ACM (New York, NY, USA, 2008), 8-14.

5. DLNA. Digital living network association. http: //www.dlna.org.

6. Finke, M., Kaviani, N., Wang, I., Tsao, V., Fels, S., and Lea, R. Investigating distributed user interfaces across interactive large displays and mobile devices. In Proceedings of the International Conference on Advanced Visual Interfaces, AVI '10, ACM (New York, NY, USA, 2010), 413-413.

7. Greenberg, S., Marquardt, N., Ballendat, T., Diaz-Marino, R., and Wang, M. Proxemic interactions: the new ubicomp? interactions 18 (January 2011), $42-50$.

8. Hall, E. T. The Hidden Dimension. Doubleday, 1966.

9. Hosio, S., Jurmu, M., Kukka, H., Riekki, J., and Ojala, T. Supporting distributed private and public user interfaces in urban environments. In Proceedings of the Eleventh Workshop on Mobile Computing Systems \& Applications, HotMobile '10, ACM (New York, NY, USA, 2010), 25-30.

10. ISO. Upnp iso standard. http://www.iso.org/iso/ catalogue_detail ?csnumber $=52674$.

11. Jenabi, M., and Reiterer, H. Primitive interaction tasks for multi-display environments (prime): a hands-on approach. In Proceedings of the International Conference on Advanced Visual Interfaces, AVI '10, ACM (New York, NY, USA, 2010), 412-412.
12. Keränen, J., Bergman, J., and Kauko, J. Gravity sphere: gestural audio-tactile interface for mobile music exploration. In Proceedings of the 27th international conference on Human factors in computing systems, CHI '09, ACM (New York, NY, USA, 2009), 1531-1534.

13. Kukka, H., Patino, R., and Ojala, T. Ubirockmachine: a multimodal music voting service for shared urban spaces. In Proceedings of the 8th International Conference on Mobile and Ubiquitous Multimedia, MUM '09, ACM (New York, NY, USA, 2009), 9:1-9:8.

14. Lorenz, A., De Castro, C. F., and Rukzio, E. Using handheld devices for mobile interaction with displays in home environments. In Proceedings of the 11th International Conference on Human-Computer Interaction with Mobile Devices and Services, MobileHCI '09, ACM (New York, NY, USA, 2009), 18:1-18:10.

15. Miller, F., Vandome, A., and McBrewster, J. Digital Ecosystem. Alphascript Publishing, 2010.

16. Newman, M. W., Elliott, A., and Smith, T. F. Providing an integrated user experience of networked media, devices, and services through end-user composition. In Proceedings of the 6th International Conference on Pervasive Computing, Pervasive '08, Springer-Verlag (Berlin, Heidelberg, 2008), 213-227.

17. O'Hara, K., Lipson, M., Jansen, M., Unger, A., Jeffries, $\mathrm{H}$., and Macer, P. Jukola: democratic music choice in a public space. In Proceedings of the 5th conference on Designing interactive systems: processes, practices, methods, and techniques, DIS '04, ACM (New York, NY, USA, 2004), 145-154.

18. Rekimoto, J. Pick-and-drop: a direct manipulation technique for multiple computer environments. In Proceedings of the 10th annual ACM symposium on User interface software and technology, UIST '97, ACM (New York, NY, USA, 1997), 31-39.

19. Sprague, D., Wu, F., and Tory, M. Music selection using the partyvote democratic jukebox. In Proceedings of the working conference on Advanced visual interfaces, AVI '08, ACM (New York, NY, USA, 2008), 433-436.

20. Turunen, M., Kallinen, A., Sànchez, I., Riekki, J., Hella, J., Olsson, T., Melto, A., Rajaniemi, J.-P., Hakulinen, J., Mäkinen, E., Valkama, P., Miettinen, T., Pyykkönen, M., Saloranta, T., Gilman, E., and Raisamo, R. Multimodal interaction with speech and physical touch interface in a media center application. In Proceedings of the International Conference on Advances in Computer Enterntainment Technology, ACE '09, ACM (New York, NY, USA, 2009), 19-26. 